\title{
La stratégie militaire à l'heure des NTIC et du « Big Data » : quelles hypothèses structurantes?
}

\author{
Par Didier Danet \\ École Spéciale Militaire de Saint-Cyr
}

\section{Résumé}

Le développement des NTIC et des méthodes d'analyse et de décision capables de traiter de gros volumes de données de terrain donne aux organisations civiles ou militaires l'opportunité de fonder leurs décisions sur une représentation fidèle de leur environnement mais crée une forme de dépendance essentielle à l'égard de ces systèmes d'information et de commandement. Il en résulte que l'analyse et l'exploitation des différentes sources de vulnérabilité dont ils sont affectés crée un nouveau champ de conflictualité qui n'obéit pas nécessairement aux principes stratégiques établis dans d'autres espaces stratégiques, qu'ils soient conventionnels ou nucléaires. Une réflexion doit donc être conduite en vue de définir ces principes stratégiques plus ou moins inédits et l'architecture institutionnelle au sein de laquelle ils devront être mis en œuvre. Il s'agit ici de réfléchir à trois idées fortes qui structurent le discours majoritaire autour de la cyber-conflictualité.

(c) 2013 Lavoisier SAS. All rights reserved Mots clés : numérisation du champ de bataille, cyber-conflictualité, continuum sécurité défense, asymétrie, attaque et défense.

\section{Abstract}

How can the military cope with the revolution of «big data»? Recent developments in ICT and Software industry give to civil and military organizations the opportunity to take decisions based on "big data" coming from the "internet of things". Thanks to this new capability, commanders in chief may almost reach the ultimate fantasy of military strategy: overcome the fog of war. But, this opportunity cannot be isolated from an increased dependency toward these information systems. And from there comes a threat or, at least, a vulnerability of modern armed forces. The strategic principles have to be revisited and, perhaps, redesigned. This paper examines three of the mainstream ideas in matter of Cyber warfare.

(c) 2013 Lavoisier SAS. All rights reserved

Keywords: digitalization of the battlefield, cyberwar, asymetry, cyberattack. 


\section{Introduction}

Soulignant la difficulté de définir simplement la notion d'analyse décisionnelle, Nicolas Menguy et Romain Zerbib la caractérisent par deux traits complémentaires. Le premier est «le maniement [par un acteur stratégique] de grandes quantités de données brutes, le plus souvent quantitatives, en vue de dresser une représentation fidèle et objective de son environnement stratégique ». Le second tient à l'adossement de ces traitements de masse à des technologies sans cesse plus performantes et « qui permettent d'automatiser le rapatriement et la centralisation d'informations depuis les points de contact informationnel de l'organisation avec son environnement ».

Ce faisant, les auteurs mettent en exergue deux des tendances fondamentales relevées par Jacques Bughin, Michael Chui et James Manyka qui, dans une livraison de $2010 \mathrm{du}$ Mc Kinsey Quaterly recensaient dix facteurs de changement induits par les transformations technologiques en ce qui concernait la structure et les activités des entreprises (Bughin, Chui, et Manyika, 2010). Si l'on s'autorise à regrouper ces dix tendances en trois grands domaines, l'étude mettait en évidence le fait que Clouds, Big Data et Smarts Assets appelaient de la part des dirigeants une réflexion approfondie quant à la nature et aux frontières de leurs organisations ${ }^{1}$, aux possibilités offertes par les capacités de traitement de volumes importants de données ${ }^{2}$ ou au renouvellement des business models conventionnels, singulièrement des modes de financement croisés entre certaines activités.

Au-delà des seules entreprises, les questions ouvertes par la double évolution des technologies de l'information et de la communication et des méthodes d'analyse adaptées à des volumes d'informations croissants et de nature hétérogène se posent à l'ensemble des organisations marchandes ou non, civiles ou militaires, privées ou publiques. Toutes sont concernées par l'influence que cette double évolution exerce sur leurs structures, leur fonctionnement, et leur niveau de performance.

S'agissant du cas particulier des organisations militaires, la question qui fait l'objet de cet article renvoie à deux aspects complémentaires et indissociables des transformations en cours dans le monde de la Défense.

Dans un sens restreint, en débat depuis longtemps, se pose la question de l'évolution des systèmes d'information et de commandement et de la numérisation du champ de bataille. C'est ainsi que le programme Scorpion de l'armée de Terre française, lancé en 2005, s'inscrit dans la logique d'une amélioration de l'efficacité des hommes et des systèmes d'armes par la mise en réseau des systèmes d'information et des systèmes de combat. L'ambition est bien d'interconnecter les différentes composantes d'un groupement tactique interarmes de sorte que les masses de données recueillies dans l'ensemble de la bulle aéroterrestre par les différents capteurs soient traitées globalement et en temps réel afin de réduire le brouillard

\footnotetext{
${ }^{1}$ Nous regroupons dans ce premier domaine les tendances relatives à l'ouverture des organisations sur un environnement globalisé au sein duquel elles peuvent solliciter des ressources à titre provisoire, ce qui implique une réticularisation poussée de l'organisation, une décentralisation très large des initiatives managériales et une capacité de co-création largement distribuée au delà des frontières de la firme. ${ }^{2}$ Ce deuxième domaine porte sur les modifications de l'environnement décisionnel. Les capacités de traitement de l'information autorisent une connaissance plus fine des paramètres de la décision et permettent en particulier d'exploiter au mieux les gros volumes de données découlant de la généralisation des objets communiquants (« internet of things»)
} 
entourant la décision du chef militaire et de surmonter les frictions dans la mise en œuvre de cette décision (Direction Générale de l'Armement, [s. d.]). La question posée est alors d'abord de nature technique : Quels types de données peut-on agréger? Quels besoins en bande passante sur le champ de bataille? Quels protocoles de communication entre les matériels ? Elle est ensuite organisationnelle et humaine : Quel impact sur le leadership ? Quelles priorités en matière d'équipements? Elle est enfin tactique : Comment modifier les manières d'opérer pour tirer parti des nouvelles possibilités de communication et d'information? L'acuité de la question de la numérisation est encore renforcée par l'apparition et le développement des robots sur le champ de bataille (Danet, Hanon, et de Boisboissel, 2012). Ceux-ci sont porteurs d'une multiplication des volumes de données introduits dans les systèmes d'information et de commandement.

Dans un sens plus large, la question des systèmes d'aide à la décision et du Big Data renvoie à l'interconnexion généralisée des systèmes militaires de traitement automatisé de l'information et à leur ouverture multiformes sur le monde civil en termes d'équipement, de réseaux, de langages d'exploitation. Le terrain de la réflexion n'est plus seulement celui des systèmes d'information et de commandement mais soulève, plus largement, la question de l'émergence d'un nouvel espace de la conflictualité, d'un champ de confrontation à part entière et dont les caractéristiques ou les principes structurants ne sont pas nécessairement les mêmes que ceux prévalant dans les espaces plus traditionnels de la conflictualité ${ }^{3}$. L'émergence rapide de ce qu'il convient d'appeler la « cyberconflictualité » soulève des questions multiples de nature technique bien évidemment mais surtout stratégiques et politiques. C'est dans ce cadre élargi que la généralisation du Big Data et de l'analyse décisionnelle emporte les conséquences les plus conséquentes en matière de réflexion stratégique.

L'influence significative qu' exercent les transformations en cours dans le « cyberespace » (Shakarian, Shakarian, et Ruef, 2013a) se mesure concrètement à l'évolution rapide des structures de commandement aussi bien que des documents doctrinaux (Ventre, 2012). Il suffit pour s'en convaincre de voir l'importance majeure prise par la chaîne du « Cybercommand » au sein du ministère de la Défense des États-Unis, voire, plus largement, dans l'ensemble des structures opérationnelles consacrées à la Défense et la Sécurité de ce pays (Bullock, Haddow, et Coppola, 2013). À un moindre degré, un mouvement comparable se dessine dans notre pays (Coustillière et Gallant, 2012). Du point de vue des doctrines, il est possible de relever qu'en France, le Livre Blanc sur la Défense de 2013 fait de la « cyberdéfense » un « enjeu mondial et une priorité nationale » là où celui de 2008, déjà considéré comme innovateur sur ce point, n'en posait que les « prodromes » (Merchet, [s. d.]). En quelques années, l'analyse décisionnelle, telle que définie par Nicolas Menguy et Romain Zebib, a donc manifestement produit des effets substantiels sur les organisations militaires et les doctrines de défense.

La chose n'est pas pour surprendre si l'on considère que toute évolution technologique redistribue plus ou moins les cartes de la puissance politico-militaire et les conditions de l'action des forces armées (Feil, 2012). Le lien entre l'état de la technologie, la structure des forces et les doctrines d'emploi est au moins aussi ancien que l'histoire militaire ellemême. De l'apparition de l'arbalète à celle de l'arme nucléaire, toutes les grandes révolutions techniques ont ouvert le champ des opportunités et des menaces à des acteurs plus ou moins

\footnotetext{
${ }^{3}$ Voir l'excellente synthèse de : (Gervais, 2012)
} 
habiles à les saisir. Que l'on songe à l'emploi de l'arme blindée durant la Seconde guerre mondiale (alors même qu'elle était connue depuis presque trente ans) et à la différence de conception ayant présidé à sa mise en œuvre entre les États-Majors français et allemand. Il n'est donc pas étonnant que les révolutions matérielles et logicielles survenues dans le domaine global des NTIC soient à l'origine d'une réflexion doctrinale sur les changements à apporter à la stratégie de Défense dans l'ensemble des pays qui maîtrisent les ressorts techniques de cette évolution (Huyghe, 2012).

La réflexion est d'autant moins surprenante que, s'ajoutant à une sorte de « loi générale » qui unirait au fil du temps progrès technique et réflexion stratégique, la présente transformation touche précisément à l'un des concepts fondamentaux de la pensée stratégique, l'imprévisibilité de la guerre appréhendée au travers des deux notions clausewitziennes de brouillard et de friction (Desportes, 2001). Qu'il s'agisse du brouillard entourant la décision du chef militaire qui ignore souvent nombre de paramètres essentiels de l'environnement, de la situation ennemie voire de la situation amie ou qu'il s'agisse des frictions résultant des inévitables impondérables dans la mise en œuvre des plans stratégiques les mieux conçus, on conçoit facilement l'attrait que peut exercer sur les États-Majors du monde entier la perspective de disposer d'un ensemble d'outils d'information, de décision et de commandement capable d'opérer un traitement automatisé des données captées par les différentes unités sur le terrain afin d'en tirer une représentation fidèle et instantanée de l'environnement du champ de bataille. Brouillard et frictions pourraient être réduits à leur plus simple expression, réalisant simultanément les rêves de Sun Ze (réduire le brouillard de la guerre par la maîtrise du renseignement) et de Schlieffen (éliminer les frictions par une planification centralisée, détaillée et rigide). Cet attrait pour des technologies de l'information capable d'atténuer, voire de faire disparaître l'imprévisibilité de la guerre est d'ailleurs une des constantes les mieux établies durant les dernières décennies des politiques de défense dans les pays qui disposent de l'avantage technologique, en premier lieu les États-Unis. Elle était déjà présente au cœur des doctrines du « Network Centric Warfare » et de la «Revolution in Military Affairs » et les progrès récents n'ont fait que ressusciter les espoirs déçus de ceux qui, comme Arquilla et Ronfeldt, avaient dû constater les limites de l'incontestable domination technologique américaine dans les combats contre-insurrectionnels en Irak ou en Afghanistan (Arquilla et Ronfeldt, 1997; Arquilla et Ronfeldt, 2001). Par de nombreux aspects, la confrontation dans le champ nouveau qu'est le cyberespace s'inscrit sans difficulté dans le cadre de la pensée stratégique occidentale, américaine en particulier : fort enracinement dans l'avance technologique, contribution significative à la quête de l'info-dominance, absence d'engagement de forces sur le terrain avec les risques de pertes qui lui sont associés, intégration d'acteurs publics et privés dans un même effort de défense et de sécurité, primauté du renseignement et de l'action préventive.

Mais, l'expérience montre que, en dépit des formidables progrès réalisés dans les équipements ou les systèmes d'armes, l'imprévisibilité de la guerre a vocation à demeurer et que le brouillard et les frictions, loin de se dissiper ou de disparaître entièrement, se déplacent et se reconstituent en d'autres points de sorte que la technologie n'est jamais la réponse définitive à l'imprévisibilité de la guerre et que la poursuite de la quête du Graal technologique peut aboutir à une approche déséquilibrée dans laquelle les économistes verraient un mauvais ajustement de la fonction de production de force, le facteur de sophistication technologique étant excessivement privilégié au détriment du facteur humain tout autant 
que du nombre des matériels disponibles (Desportes, 2009). Les déboires associés à cette « tentation technologique » doivent donc guider les responsables des organisations militaires à réfléchir à ce qu'il convient d'attendre de la généralisation de l'analyse décisionnelle ou du Big Data. Sont-ils de nature à modifier fondamentalement les cadres habituels de la pensée stratégique? Les doctrines existantes, par exemple l'action globale des forces, doivent-elles être repensées profondément? La numérisation du champ de bataille doit-elle bénéficier d'une priorité dans les efforts consentis en faveur de la Défense? L'espace nouveau de conflictualité qu'est le « cyber » doit-il être pensé différemment en termes stratégiques?

C'est à ce niveau que se posent les questions les plus complexes et les plus sensibles de la réflexion stratégique tant les incertitudes sont encore grandes. Il serait vain de vouloir les épuiser dans le cadre de cette communication tant elles appellent de développements entremêlant les sciences de l'ingénieur aussi bien que les sciences humaines, politiques et sociales. Aussi, notre propos se limitera à l'examen de trois idées qui semblent s'imposer dans le champ de la réflexion stratégique et qui nous paraissent mériter un regard critique et une discussion de fond. Il nous semble en effet que ces trois idées structurantes, souvent présentées comme des postulats de la réflexion stratégique contemporaine, comportent tout à la fois une part de réalité mais aussi de mythe et que cette dernière dimension est préjudiciable à la formulation de doctrines répondant aux enjeux du moment.

\section{Trois hypothèses structurantes de la réflexion stratégique dans le cyberespace}

De la lecture des principaux auteurs qui forment le courant majoritaire de la réflexion stratégique dans le cyberespace se dégagent trois idées que leur acceptation générale semble élever au rang de postulats. Ces trois hypothèses tiennent respectivement aux frontières du conflit dans le cyberespace, à la figure de ce conflit et à son issue possible.

\subsection{Le continuum sécurité / défense}

La généralisation des NTIC et de l'analyse décisionnelle dans le monde militaire s'est traduite par le développement de systèmes de commandement et de contrôle intégrés, capables de tirer parti de la masse d'informations recueillies par l'ensemble des dispositifs ad hoc jusqu'au niveau tactique le plus élémentaire, celui du soldat sur le terrain qui joue incontestablement le rôle d'un capteur communiquant, lequel transmet aujourd'hui des données de toute nature et sous des formes multiples. De la même manière que l'internet des objets (Internet of things) est une source essentielle de développement du Big Data pour les organisations civiles, l'Internet of soldiers est un facteur de transformation essentiel des systèmes de décision militaire. La collecte et le traitement automatisé d'un volume sans cesse croissant de données de terrain produites tant par les hommes que par les équipements et systèmes d'armes spécialisés ou non est de nature à dissiper une partie du brouillard et des frictions de la guerre, réduisant ainsi l'ignorance relative qui entoure le processus de décision du chef au combat, et à lui permettre d'accélérer le rythme de la boucle OADA, ce qui lui confère un avantage certain sur un adversaire qui ne disposerait pas de systèmes de commandement et de contrôle aussi performants. Une meilleure perception de la réalité du terrain serait la garantie d'une efficacité accrue des armées les plus avancées. 
Mais, en contrepartie de ce surcroît d'efficacité, l'interconnexion généralisée de dispositifs automatisés de traitement de l'information et la mise en place de réseaux qui sont nécessairement ouverts sur le monde extérieur constituent une forme de vulnérabilité en ce sens que, compte tenu du fait que l'avantage militaire de ces armées dépend de la supériorité de leurs systèmes d'information et de commandement, les atteintes portées à ces systèmes sont de nature à dégrader, voire à interdire, l'action de l'ensemble des forces qui leur sont asservies. Or, à cet égard, les composantes physiques et logicielles des systèmes militaires sont bien souvent dérivées très directement des systèmes civils et elles leur sont nécessairement reliées (Bonnemaison, 2013). En dehors de consoles spécifiques qui équipent certains systèmes d'armes, les ordinateurs utilisés par les forces sont très proches de ceux utilisés dans le monde civil. Les systèmes de géolocalisation des matériels militaires ne sont pas très éloignés de ceux qui sont fournis aux entreprises pour les besoins de leurs activités logistiques. Les réseaux de transmission de l'information sont pour partie communs avec ceux du monde civil.

Les systèmes de commandement et de contrôle des forces armées sont donc sujets à des menaces dont la nature et les formes sont les mêmes que celles qui pèsent sur l'ensemble des organisations civiles, administrations ou entreprises notamment (Andreasson, 2011 ; Betz et Stevens, 2011). La dépendance des systèmes de commandement et de contrôle ainsi que des systèmes d'armes à l'égard de dispositifs techniques et de méthodes de décision qui sont très largement partagés entre la Défense et les acteurs du monde civil efface les frontières traditionnelles entre les questions de sécurité et les questions de défense, entre le monde des armées et le monde de l'entreprise, entre les acteurs publics et les acteurs privés. Le cyberespace apporte la touche finale à un continuum sécurité / défense qui n'est certes pas nouveau puisqu'il constituait l'un des principes fondamentaux de l'ordonnance de 1959, mais il réduit de manière sans doute inédite le champ spécifique de l'action militaire et en banalise certains des aspects dimensionnant.

Une première inflexion majeure de la pensée stratégique dans un monde caractérisé par le développement des NTIC et de l'analyse décisionnelle est la notion de « continuum » qui invite à penser les questions de sécurité et de défense simultanément et globalement. Sont donc ainsi dépassées les lignes de clivage habituelles qui, au-delà d'un espace commun relativement limité conduisait chaque institution à disposer d'un champ de compétence et de responsabilité particulier, créant une spécificité en termes de questionnements, d'institutions, de métier, de cadre juridique. L'extension spectaculaire de cet espace commun que traduit la notion de continuum modifie radicalement les cadres de référence les mieux établis (doctrinaux, institutionnels, professionnels...) et engendre un processus de recomposition donc certains traits saillants se dégagent de plus en plus nettement : primauté du renseignement, rapprochement des métiers policiers et militaires, rôle moteur de certaines institutions particulièrement bien placée au regard de la notion de continuum, à l'exemple de la gendarmerie (Hesbrard et Ferry, 2012) intégration d'un ensemble d'acteurs hétérogènes dans le nouvel appareil de défense et de sécurité (Samaan, 2008).

\subsection{L'asymétrie renforcée des conflits à l'ère numérique}

Pour les États et les forces armées qui en émanent, les opportunités et menaces qui résultent des NTIC ou du développement du Big Data élargissent substantiellement le champ des possibles en matière de conflits de basse intensité et nécessitent de mettre en 
place les stratégies et moyens nécessaires de la lutte informatique défensive et offensive. Celle-ci permet, en effet, d'exercer des effets gradués (de la simple gêne à la destruction d'équipements ou d'installations physiques et à la paralysie des forces adverses) sur des cibles variées (militaires ou civiles) et sous des formes multiples (ouvertes ou masquées notamment). Elle offre donc de nouveaux leviers pour mener des opérations militaires conventionnelles, (Baud, 2012; Arquilla, 2011) peser sur la volonté adverse et infléchir le comportement d'un État hostile (Lifland, 2012; Manson, 2011). À n'en pas douter, la lutte informatique offensive et défensive sera l'un des éléments privilégiés des affrontements futurs, qu'ils soient de basse ou de haute intensité.

Mais, le recours à des techniques de ce type ne sera pas réservé à des acteurs étatiques. Tout semble réuni pour que le « ticket d'entrée » dans le champ de la conflictualité cybernétique soit accessible à de nombreux groupes de nature diverse (Shakarian, Shakarian, et Ruef, 2013b), voire à des individus isolés. Certaines formes d'agression sont assez peu exigeantes en matière de capital intellectuel ou financier et peuvent donc s'envisager à coût réduit, devenant dès lors accessibles à un grand nombre de mouvements politiques, de groupes armés et leur ouvrant la possibilité de peser sur des organisations qu'ils ne pourraient envisager d'affronter sur un autre terrain (Manjikian, 2010). La dépendance des forces armées (et plus généralement des autres organisations critiques du monde civil) à l'égard de systèmes de traitement de l'information nécessairement ouverts sur l'extérieur engendre une égalisation sans doute inédite des acteurs de la conflictualité.

La réflexion stratégique doit donc poursuivre et approfondir la relecture rendue nécessaire par la généralisation des conflits asymétriques. Le fait que des forces armées disposant d'une absolue supériorité technologique aient été mises en échec de manière récurrente dans les conflits irakien, afghan ou libanais (Goya, 2009; Goya, 2007) a montré les limites de cette forme de supériorité et imposé la réintroduction dans le paysage de la réflexion stratégique les concepts issus de l'expérience des guerres contre-insurrectionnelles (Vallons, 2010). Si l'on admet que les affrontements à venir dans le champ de la cyber-conflictualité se caractériseront par une exacerbation de cette asymétrie, nul doute que la seule réponse fondée sur la recherche (et éventuellement la maîtrise) de l'info-dominance ne sera pas suffisante.

\subsection{La supériorité de l'attaque sur la défense}

Enfin, des caractéristiques précédentes découle un dernier trait fondamental pour la réflexion stratégique dans le contexte envisagé ici, la supériorité de l'attaque sur la défense. Dans la dynamique toujours renouvelée de l'attaque et de la défense, la lutte informatique semble définitivement faire pencher le fléau de la balance du côté de la première. Mobilisant des moyens limités, un acteur isolé y apparaît capable de peser sur la volonté d'un État puissant en lui infligeant, de manière éventuellement anonyme, des dommages apocalyptiques, par exemple, au travers de l'atteinte à des infrastructures critiques. L'attaque présente donc des traits particuliers (dissimulation, soudaineté, effets, etc.) susceptibles de rendre inopérantes les mesures de prévention, de protection ou de riposte qui auront été mises en place. Son auteur peut se réfugier dans un anonymat difficile à percer, voire faire porter par d'autres la responsabilité de ses actes (Healey, 2011). Il peut implanter une menace qui ne sera activée que dans un futur plus ou moins proche ou dans des conditions particulières. Il dispose d'un nombre 
non limité de cibles potentielles dont les points de liaison avec le monde extérieur sont souvent innombrables. Bref, toute politique de sécurité et de défense est presque inexorablement vouée à l'échec pour autant qu'elle succombe sous son propre poids en termes de complexité, de coûts et de délais de toute sorte. Il importe que chaque acteur dont l'action dépend de systèmes de traitement automatisé de se prémunir contre les agressions susceptibles de le frapper. Il importe surtout pour les acteurs étatiques et les forces armées au premier chef, d'exploiter les opportunités offertes par la lutte informatique offensive. Le premier des domaines concernés est certainement le renseignement. En amont de l'action visant à gêner, paralyser ou détruire des systèmes adverses, les politiques nationales de sécurité et de défense tendent à l'intégration du renseignement civil et militaire au sein d'appareils de coordination et de commandement dont les équilibres institutionnels varient d'un pays à l'autre. La primauté du renseignement découle directement de la nécessité de prévenir une attaque à laquelle il serait difficile de faire face ou de riposter. De même, l'évolution récente des discours officiels qui reconnaissent désormais la légitimité (sous certaines conditions) de la lutte informatique offensive peut être considérée comme la conséquence de cette supériorité de l'attaque sur la défense.

La troisième caractéristique majeure de la réflexion stratégique est donc de considérer que la supériorité de l'attaque doit conduire à donner une place centrale à la prévention et à développer en conséquence des appareils de renseignement affranchis des traditionnelles barrières qui le caractérisaient. Un autre trait caractéristique est l'évolution de la doctrine concernant la lutte informatique offensive, naguère encore considérée comme indésirable et dont les pays qui disposent de la maîtrise de l'information envisagent la mise en œuvre de plus en plus ouvertement.

\section{Conclusion de la première partie}

Les trois idées évoquées dans la première partie n'épuisent manifestement pas les questions qui se posent à la réflexion stratégique dans un monde caractérisé par l'évolution rapide des données technologiques ainsi que la généralisation de l'analyse décisionnelle dans les organisations civiles et militaires. Elles nous semblent cependant importantes par le caractère déterminant de leur influence sur la conception générale de la conflictualité contemporaine et les conséquences qu'elles entraînent pour l'action globale des forces armées et, plus généralement, de l'ensemble des acteurs qui s'inscrivent dans le continuum sécurité / défense

\section{Discussion des trois hypothèses.}

La question qui se pose alors est celle de la pertinence de ces trois idées structurantes sur lesquelles s'appuierait une nouvelle approche stratégique des questions de sécurité et de défense à l'ère de la cyber-conflictualité. Bien qu'elles fassent l'objet d'un assez large consensus, elles méritent certainement d'être revisitées de manière régulière, ne serait-ce qu'au regard des transformations incessantes de l'univers technologique aussi bien que du contexte géo-stratégique, des équilibres institutionnels, de l'environnement socio-écono- 
mique... De fait, si les cadres de la réflexion ainsi présentés apparaissent cohérents dans leurs principes, certaines de leurs modalités, de leurs conséquences ou des enseignements que l'on en tire peuvent être sujets à débat et à réflexion.

\subsection{Quelle réponse institutionnelle au continuum sécurité / défense?}

Le fait que les systèmes de commandement et de contrôle ainsi que les systèmes d'armes soient soumis à des menaces de même nature que les systèmes civils appelle une réflexion et une réponse qui semble «naturellement » procéder d'une coopération générale des acteurs concernés : forces armées, police, administration, entreprises. Ce besoin de coopération, de coordination ou d'articulation d'acteurs hétérogènes, notamment issus des secteurs public et privé, est tout sauf nouveau. Il est même au cœur des nouvelles pratiques du management public à travers la multiplication des partenariats public privé. S'agissant du cyberespace, un certain nombre de partenariats sont déjà en place ou sont en projet afin de faire face à certaines vulnérabilités qui sont connues, par exemple, la dépendance à l'égard de fournisseurs étrangers pour des éléments critiques de la chaîne de traitement de l'information, qu'il s'agisse des composants matériels, des systèmes de géolocalisation, des logiciels mis en œuvre, des espaces de stockage des données. Le fait de substituer une entreprise nationale à un fournisseur étranger, éventuellement issu d'un pays potentiellement hostile, peut apparaître comme une mesure de bon sens. Encore convient-il de s'appuyer sur un ensemble cohérent de filières industrielles (Tissier, 2012) sur les bons acteurs (techniquement efficaces, économiquement compétitifs, indépendants et pérennes) et de mettre en place les bons outils de coordination. Sous ces deux critères et au vu de l'expérience acquise en matière de partenariats public-privé qui sont la forme aujourd'hui privilégiée de coordination entre l'administration et les entreprises, la mise en place de dispositifs contractuels comme réponse à la continuité de la menace appelle sans doute une réflexion approfondie et des mécanismes d'engagement réciproques enrichis (Lobel, 2012). Il convient de ce point de vue de ne pas oublier que la contractualisation des relations entre l'État et des prestataires privés est loin d'être la seule forme de coordination envisageable et que, face à la complexité et à l'hétérogénéité des questions soulevées, il serait opportun d'envisager une panoplie complète de solutions coopératives allant de la relation contractuelle pure et simple jusqu'à l'intégration d'ensembles d'activités au sein de structures étatiques en passant par la réglementation ou la mise en place de mesures incitatives. Le danger serait alors que la réflexion stratégique soit intellectuellement contrainte par des dogmes, ceux du Nouveau Management Public, qui ont conduit à des déboires connus en termes d'efficacité et de contrôle de l'action des forces armées.

\subsection{Mythes et réalité du pouvoir égalisateur du cyberespace}

Le pouvoir égalisateur de la technologie face à la vulnérabilité des appareils étatiques les plus puissants est l'un des thèmes les plus anciens de la littérature dans ce domaine. Il peut d'ailleurs se présenter sous deux formes opposées. La première, positive, consiste à créditer de petits groupes d'activistes en lutte contre des régimes dictatoriaux d'une capacité d'influence et d'entrainement qui fait d'eux les véritables moteurs des « révolutions 2.0 ». 
Dans ce cas, c'est moins par l'atteinte à des systèmes de commandement et de contrôle ou à des infrastructures critiques que passe l'action des ces mouvements que par l'exploitation des nouveaux moyens de communication à des fins de propagande et de mobilisation des foules ou de l'opinion publique mondiale (Bazan et Varin, 2012). La question est d'ailleurs vivement discutée (Béchir Ayari, 2011) et elle n'est pas directement celle qui nous occupe ici. La seconde facette de l'hyper-asymétrie, négative, est celle qui place les pays les plus avancés dans une situation de vulnérabilité critique face à des individus ou des groupes qui, pour des motivations variables, politiques ou criminelles notamment (Kshetri, 2005) menacent de conduire des actions de type terroriste frappant la population à travers des attaques menées contre des infrastructures critiques : installations nucléaires, usines chimiques, réseaux d'alimentation en eau, infrastructures de transport ferroviaire ou aérien.

Sans contester la gravité de la menace, il semble toutefois que les retours d'expérience montrent toute la complexité et tout le coût de telles attaques. Ainsi, pour ce que l'on peut savoir du cas Stuxnet, qui correspond typiquement à la dégradation d'un système de télégestion d'une installation industrielle par le biais d'une intrusion informatique, les moyens à mobiliser pour parvenir au résultat souhaité (provoquer des dysfonctionnements dans une centrale nucléaire iranienne) sont d'une exceptionnelle ampleur (Shakarian, Shakarian et Ruef, 2013c; Gregory, 2011). Concevoir un ver capable de tromper le système d'exploitation en exploitant plusieurs de ses failles restées jusque là inédites, puis d'échapper aux logiciels anti-virus les plus performants et de reprogrammer des automates industriels d'un constructeur donné sans que les anomalies ainsi provoquées ne soient rapidement détectées et traitées (Faillière, O Murchu et Chien, 2011), enfin, parvenir à l'implanter dans le système visé, tout cela nécessite de mobiliser des ressources humaines et des moyens opérationnels tout à fait extraordinaires. Il convient en premier lieu de mettre au point le virus, ce qui suppose une équipe de spécialistes représentant selon toute vraisemblance plusieurs dizaines de personnes et plusieurs mois de travail, l'équipe concernée devant notamment comporter des spécialistes maîtrisant parfaitement le système d'exploitation et le dispositif industriel visé. Dans la phase de mise en œuvre, l'équipe doit pouvoir s'approprier frauduleusement des certificats d'authentification permettant de tromper les mesures de sécurité informatiques. Elle doit également disposer d'informations opérationnelles sur le site et l'installation technique qui sont visés afin de le pénétrer pour implanter le virus. Sans aller jusqu'à dire que ce type d'opération ne peut en aucun cas être le fait d'individus isolés ou de groupes criminels ou terroristes, les compétences à réunir aussi bien que les moyens à mettre en œuvre semblent davantage du ressort d'appareils étatiques constitués à cette fin (Markoff et Sanger, 2011). Le champ de la cyber-conflictualité ne serait donc pas nécessairement caractérisé par une asymétrie exceptionnellement marquée entre les acteurs. La menace d'une action terroriste de premier plan conduite par un petit groupe d'individus à l'encontre d'une infrastructure critique d'un grand pays développé n'est donc peut-être pas à mettre au premier rang des priorités de la réflexion stratégique en matière de cyberconflictualité.

\subsection{Relativiser la supériorité de l'attaque sur la défense}

De nombreux auteurs mettent en exergue l'avantage pris par l'attaquant sur le défenseur dans l'hypothèse de conflits survenant dans le cyberespace ainsi que l'incapacité du défenseur à prévenir et enrayer les effets destructeurs de l'initiative adverse, voire à 
riposter à cette dernière lorsqu'elle s'est produite. Pour illustrer cette rupture de l'équilibre entre attaque et défense, des spécialistes ou responsables politiques évoquent volontiers la menace d'un « cyber Pearl Harbor » ou d'un « cyber-9/11 », ce qui illustre parfaitement l'idée d'une attaque soudaine, brutale et aux conséquences apocalyptiques. Ainsi, dans l'ouvrage de Richard Clarke, l'attaque menée contre les États-Unis provoque l'explosion d'usines chimiques libérant des nuages de gaz mortels, le sabotage du contrôle aérien et la vague de catastrophes aériennes qui en résulte, la paralysie des centrales électriques qui prive le pays d'énergie et de lumière (Clarke et Knake, 2012; Brenner, 2011). Si, sous les réserves évoquées précédemment, l'hypothèse d'une attaque de grande ampleur visant à la destruction d'infrastructures critiques (réseaux bancaires, installations industrielles de type Seveso, lignes de transport...) ou à la paralysie effective de systèmes d'armes ou de commandement n'est pas exclue, une expérience comme celle de l'Estonie montre qu'il n'y a pas d'automaticité dans la réussite de l'attaque et dans les effets destructeurs qu'elle s'assigne. Les politiques de prévention et de protection ne sont pas par nature vouées à l'échec (MacKinnon, Bacon, Gan, Loukas, Chadwick, et Frangiskatos, 2013). Ainsi, dans le cas estonien qui est sans doute l'un des plus commentés, les conditions idéales semblent réunies pour favoriser l'attaquant : un pays cible ayant fait le pari de la dématérialisation de ses processus de gestion et dès lors très dépendant du réseau internet pour le bon fonctionnement de son administration et de ses entreprises, un attaquant (supposé) disposant de tous les moyens étatiques d'une grande puissance, un mode d'action simple mais facile à mettre en œuvre, une capacité d'action dans la durée. Dans ces conditions, en supposant que la volonté de l'attaquant ait été de paralyser certaines institutions, voire de provoquer certaines formes de panique ou de désordre social, force est de constater que rien de tel ne s'est produit. ${ }^{4}$ Conduite pendant trois semaines, l'attaque n'a abouti qu'au déni de service d'un petit nombre de banques pendant une durée limitée (deux heures au plus) et en un nombre tout aussi restreint d'occasions. Dans une telle hypothèse où tout semblait favoriser l'attaquant, aucune paralysie durable n'a été constatée, aucun pirate n'a pu s'introduire dans le réseau bancaire, aucun avion ne s'est écrasé et aucun train n'a déraillé. L'impossibilité pour certains Estoniens d'accéder à leur compte bancaire en ligne pendant deux heures fait davantage songer à une panne due à un pic de fréquentation plutôt qu' aux destructions qui sont le résultat d'un affrontement militaire.

L'exemple estonien montre que l'avantage conféré à l'attaquant est sans doute surestimé par les spécialistes comme Richard Clarke (Rid, 2012; Libicki, 2011). Outre les réserves émises à propos de la capacité de groupes non étatiques de mener de telles attaques compte tenu de leur complexité et de leur coût, il apparaît également que la mise en œuvre des attaques est difficile à maîtriser compte tenu précisément de l'interconnexion généralisée de systèmes de traitement qui utilisent les mêmes briques physiques ou logicielles. L'attaquant est donc soumis à un dilemme : déployer une arme spécifique susceptible de ne frapper que la cible visée mais dont le coût de conception sera forcément beaucoup plus élevé ou utiliser une arme générique, plus facile d'accès mais susceptible d'engendrer des dommages dans des pays amis, voire dans le pays d'origine de l'attaquant. La dynamique de l'attaque et de la défense n'est pas moins facteur d'incertitude. En effet, la mise au point d'une attaque

${ }^{4}$ L'évaluation des effets de l'attaque contre l'Estonie demeure cependant un enjeu de débat. Voir par exemple, l'estimation haute de Jenik (2009) et Shackelford (2010). 
ciblée suppose un investissement et des délais significatifs tandis que la riposte une fois l'attaque découverte peut être rapide (comblement de la faille de sécurité) et l'attaque est alors définitivement enrayée. Pour être pleinement efficace dans la durée, l'attaque doit alors s'efforcer de demeurer cachée, ce qui renforce une fois encore la complexité de l'opération.

La balance entre l'attaque et la défense est-elle alors aussi systématiquement en faveur de l'attaque? Sans s'interdire de penser que la nature des opérations implique certainement un équilibre particulier de la balance des forces, il ne serait sans doute pas opportun de penser la réflexion stratégique dans un cadre où la supériorité de l'attaque serait considérée comme une donnée définitive du problème (Sommer et Brown, 2011).

\section{Conclusion}

Les données nouvelles des NTIC et de l'analyse décisionnelle conduisent à renouveler la réflexion stratégique dans le domaine militaire aussi bien que dans le domaine civil. Rien ne serait plus néfaste que de plaquer d'anciennes conceptions ou d'anciens modèles (ceux de la stratégie nucléaire par exemple) (Schutte, 2012) sur une situation qui ne s'y apparente que très partiellement. Pour autant, il ne serait sans doute pas plus pertinent de postuler le caractère strictement inédit de ce type de conflit et l'impérieuse nécessité de faire table rase de la réflexion antérieure. Or, c'est l'un des risques qui nous semble se dégager de la combinaison des trois idées structurantes qui ont été évoquées ici.

En effet, quelle est la figure de l'ennemi produite par le discours majoritaire? (Conesa, 2009; Dunn Cavelty, 2013; Conway, 2009). La combinaison continuum / asymétrie / attaque conduisent à une forme d'indistinction de l'adversaire (qui peut être un individu aussi bien qu'une puissance étatique) et qui est susceptible d'attaquer sans crainte de riposte ou de représailles les forces armées aussi bien que les intérêts vitaux ou la population d'un pays aussi bien protégé soit-il. Cette figure de l'ennemi nous semble reposer sur une vision exacerbée de la menace dans toutes ses dimensions. On ne saurait le reprocher à des responsables chargés de préserver la sécurité et la défense d'une Nation et à qui l'on reprocherait sans aucun doute de n'avoir pas imaginé le pire si une attaque devait subvenir. Mais, en même temps, cette approche exacerbée peut conduire à des décisions non optimales compte tenu de leur coût économique ou de leurs effets socio-politiques. Ainsi, faire de la cyber-défense une priorité nationale suppose dans un environnement contraint de réduire l'effort consenti pour faire face à d'autres menaces, lesquelles ne sont peut-être pas moins présentes ou réalistes que le cyber-conflit. Faire du renseignement et de la prévention des attaques la seule véritable réponse à la menace peut conduire à mettre en place des procédures peu conformes aux libertés civiles comme l'a montré encore récemment le cas américain (Douzet, Samaan et Desforges, 2009). Tirer de l'idée de continuum une nouvelle structure institutionnelle rassemblant un groupe hétérogène d'acteurs dont la coordination serait fondée sur un type restreint de mécanismes contractuels serait sans doute ignorer les difficultés déjà constatées dans des constructions de ce type.

Pour toutes ces raisons, il nous semble que la réflexion stratégique dans un univers caractérisé par les progrès des NTIC et de l'analyse décisionnelle doit se garder de deux écueils opposés : l'excès de conservatisme (qui conduirait à plaquer sur une situation nou- 
velle des schémas anciens) et le déni des précédents (qui consisterait à penser que la donne stratégique est radicalement différente de ce qui a pu exister jusqu'ici). Traditionnellement, l'écueil le plus important est l'excès de conservatisme qui conduit les forces armées à refaire la dernière guerre. En l'espèce, le risque nous semble plutôt être opposé. Cela fait de la poursuite et de l'approfondissement du débat stratégique une impérieuse nécessité pour les années à venir (Mongin, 2013).

\section{Bibliographie}

Andreasson K. J., 2011. Cybersecurity: Public Sector Threats and Responses. CRC Press Inc, Boca Raton.

Arquilla J., Ronfeldt D. F., 1997 In Athena's Camp: Preparing for Conflict in the Information Age. Rand Corporation, Santa Monica.

Arquilla J., Ronfeldt D. F., 2001. Networks and Netwars: The Future of Terror, Crime, and Militancy. Rand Corporation, Santa Monica.

Arquilla J., 2011. The Computer Mouse that Roared: Cyberwar in the Twenty-First Century. Brown J. World Aff. 18 (1), 39-48.

Baud M.,2012. La cyberguerre n'aura pas lieu, mais il faut s'y préparer. Polit. Étrangère 2, 305.

Bazan S., Varin C., 2012. Le Web à l'épreuve de la cyberguerre en Syrie. Études 417 (12), 595-606.

Béchir Ayari M., 2011. Non, les révolutions tunisienne et égyptienne ne sont pas des révolutions 2.0 . Mouvements 66 (2), 56-61.

Betz D. J., Stevens T. C., 2011. Cyberspace and the State: Towards a Strategy for Cyber-power. Routledge, London.

Bonnemaison E., 2013. Le continuum Défense - Sécurité dans le cyberespace In FIC 2013, Lille.

Brenner J., 2011. America the Vulnerable: new Technology and the Next Threat to National Security. Penguin Press, New York .

Bughin J., Chui M., Manyika J., 2010. Clouds, Big Data and Smart Assets: Ten Tech enabled Business Trands to Watch. McKinsey Quaterly.

Bullock J. A., Haddow G. D., Coppola D. P., 2013. 8 - Cybersecurity and Critical Infrastructure Protection In Bullock J. A., Haddow G. D., Coppola D. P. Introduction to Homeland Security. Butterworth-Heinemann, Boston, 283-321.

Clarke R. A., Knake R., 2012. Cyber War: The Next Threat to National Security and What to Do About It. Harper andCollins, New York.

Conesa P., 2009. La fabrication de l'ennemi. Rev. Int. Strat. 4 (76), 35-44.

Conway M., 2009 Le cyber-terrorisme. Cités 39 (3), 81-94.

Coustillière A., Gallant M., 2012 La montée en puissance de la cyberdéfense au sein des armées. Rev. Gendarm. Natl. 4, 9-16.

Danet D., Hanon J.-P., De Boisboissel G., 2012. La robotisation du champ de bataille : évolution ou robolution ? In Danet D., Hanon J.-P., De Boisboissel G. La guerre robotisée, Economica, Paris, 5-28.

Desportes V., 2001. Comprendre la guerre 2e éd. Economica, Paris.

Desportes V., 2009. Armées: technologisme ou juste technologie? Politique Étrangère 2, 403-418.

Direction Générale de l'armement. Le programme Scropion http://www.defense.gouv.fr/dga/equipement/ terrestre/le-programme-scorpion

Douzet F., Samaan J.-L., Desforges A., 2009. Les pirates du cyberespace. Hérodote 134 (3), 176-193.

Dunn Cavelty M., 2013. From Cyber-Bombs to Political Fallout: Threat Representations with an Impact in the Cyber-Security Discourse. Int. Stud. Rev. 15 (1), 105-122. 
Faillière N., O Murchu L., Chien E., 2011. W32. Stuxnet Dossier. Symantec

http://www.symantec.com/content/en/us/enterprise/media/security_response/whitepapers/w32_stuxnet_dossier.pdf > Feil J. A., 2012. Cyberwar and Unmanned Aerial Vehicles : Using New Technologies, From Espionage to Action. Case West. Reserve J. Int. Law. 45 (1/2), 513-544.

Gervais M., 2012. Cyber Attacks and the Laws of War. Berkeley J. Int. Law. 30 (2), 525-579.

Goya M., 2009. Irak : Les armées du chaos $2^{\mathrm{e}}$ éd. Economica, Paris.

Goya M., 2007. Dix millions de dollars le milicien. Politique Étrangère 1, 191-202.

Gregory D., 2011. The Everywhere War. Geogr. J. 177 (3), 238-250.

Healey J., 2011. The Spectrum of National Responsibility for Cyberattacks. Brown J. World Aff. 18 (1), 57-70.

Hesbrard J., Ferry J., 2012. Gendarmerie et cybersécurité. Rev. Gendarm. Natl. 4, 61-68.

Huyghe F.-B., 2012. Stratégie dans le cyberespace. Médium 31(2), 129-146.

Jenik A., 2009. Cyberwar in Estonia and the Middle East. Network Security 2009 (4), 4-6.

Kshetri N., 2005. Pattern of Global Cyber War and Crime: A Conceptual Framework. J. Int. Manag. 11 (4), 541-562.

Libicki M., 2011. The Nature of Strategic Instability in Cyberspace. Brown J. World Aff. 18 (1), 71-79.

Lifland A., 2012. Cyberwar. Harv. Int. Rev. 33 (4), 7-8.

Lobel H., 2012. Cyber War inc. : The Law of War Implications of the Private Sector's Role in Cyber Conflict. Tex. Int. Law J. Summer, 618-641.

Mackinnon L., Bacon L., Gan D., Loukas G., Chadwick D., Frangiskatos D., 2013. Cyber Security Countermeasures to Combat Cyber Terrorism. In Akhgar B and Yates S. Strategic Intelligence Management. Butterworth-Heinemann, London, 234-257.

Manjikian M. M., 2010. From Global Village to Virtual Battlespace: The Colonizing of the Internet and the Extension of Realpolitik. Int. Stud. Q. 54 (2), 381-401.

Manson G. P., Cyberwar: The United States and China Prepare For the Next Generation of Conflict. Comp. Strat. 30 (2), 121-133.

Markoff W. J. B., John, Sanger D. E. Stuxnet Worm Used Against Iran Was Tested in Israel. New York Times 15 janvier 2011.

http://www.nytimes.com/2011/01/16/world/middleeast/16stuxnet.html

Merchet D. Livre blanc : Otan, cyberdéfense et outre-mer en vedettes In Secret Défense http://www.marianne. net/blogsecretdefense/Livre-blanc-Otan-cyberdefense-et-outre-mer-en-vedettes_a856.html

Mongin O., 2013. Les cyberattaques, armes de guerre en temps de paix. Esprit 1, 32-49.

Rid T., 2012. Think Again: Cyberwar. Foreign Policy192, 1-11.

Samaan J.-L., 2008. Mythes et réalités des cyberguerres. Politique Étrangère 4, 829-841.

Schutte S., 2012. Cooperation beats Deterrence in Cyberwar. Peace Econ. Peace Sci. Public Policy. 18 (3), 1-11.

Shackelford S. J., 2010. Estonia Three Years Later: A Progress Report on Combating Cyber Attacks. J. Internet Law 13 (8), 22-29.

Shakarian P., Shakarian J., Ruef A., 2013a. Cyber Warfare: Here and Now In Shakarian P., Shakarian J., Ruef A. Introduction to Cyber-Warfare Syngress, Boston, 1-8.

Shakarian P., Shakarian J., Ruef A., 2013b. Cyber Attacks by Nonstate Hacking Groups: The Case of Anonymous and Its Affiliates In Shakarian P., Shakarian J., Ruef A. Introduction to Cyber-Warfare Syngress, Boston, 67-110.

Shakarian P., Shakarian J., Ruef A., 2013c. Attacking Iranian Nuclear Facilities: Stuxnet In Shakarian P., Shakarian J., Ruef A. Introduction to Cyber-Warfare Syngress, Boston, 223-239.

Sommer P., Brown I., 2011. Reducing Systemic Cybersecurity Risk. Organ. Econ. Coop. Dev. Work. Pap. No IFPWKPFGS 2011 Vol. 3. http://papers.ssrn.com/sol3/papers.cfm?abstract_id=1743384 
Tissier G., 2012. Pourquoi une politique industrielle en matière de cyber-sécurité ? Rev. Gendarm. Natl.4,93-99. Vallons G.-H. B. Des., 2010. Faut-il brûler la contre-insurrection? Choiseul Éditions, Paris.

Ventre D., 2012. Cyber Conflict: Competing National Perspectives. ISTE Ltd and John Wiley \& Sons Inc, London. 
